\section{Sugar receptor sites in the fly's tongue}

from our

Insect Physiology Correspondent

SINCE the isolation of a 'sweet-sensitive protein' from the bovine tongue there has been increasing interest in the properties of sensory receptor substances and receptor sites. Von Frisch studying the honeybee in 1935 and Dethier studying the blowfly in 1955 failed to discover configurations that were common to the stimulating carbohydrates and were absent in the tasteless ones. But their results underlined the extreme specificity of insect receptors as compared with those of mammals. Dethier observed, however, that the weakly stimulating sugar, mannose, markedly reduced stimulation by fructose but had no effect on glucose sensitivity. Evans (1961) inferred that there must be at least two different sites in the sugar receptor of the blowfly tongue, a 'glucose site' and a 'fructose site'.

Shimada et al. (J. Insect Physiol., 20, 605 ; 1974) have now re-investigated this problem in the large labellar hairs of the flesh fly Boettcherisca peregrina. In earlier work these authors had found that the sugar receptor in this fly with respect to sucrose could be modified by treatment with the sulphhydryl reagent, p-chloromercuribenzoate (PCMB). PCMB seemed to react with sulphhydryl groups in proteins of the sugar receptor, but not at the sugar-binding site; it apparently blocked a change in the permeability of the receptor membrane. Shimada et al. have now made a detailed study of the effects of PCMB on the electrophysiological response of single receptor hairs, to fructose, glucose and other sugars.

It turned out that there is a striking difference in the effect on the response to glucose and fructose: response to glucose was completely repressed; res-

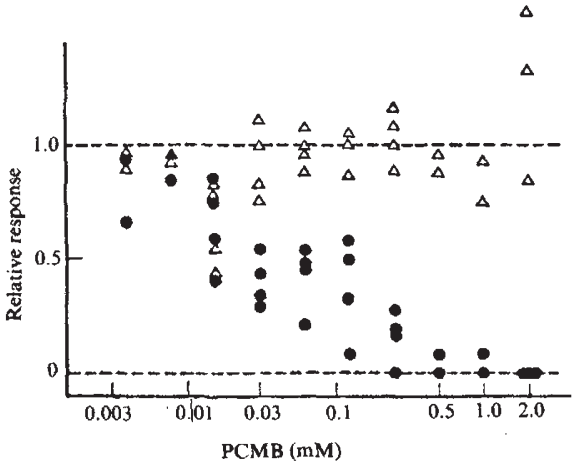

Depression of responses to fructose and to glucose by treatment with $p$ chloromercuribenzoate at graded concentrations. Ordinate: response relative to that before treatment. Duration of treatment with PCBM was always 3 min. $\triangle, 0.1 \mathrm{M}$ fructose; $0.2 \mathrm{M}$ glucose. ponse to fructose was unaffected (see figure). This constitutes the first direct demonstration of the existence of a 'glucose site' and a 'fructose site' as suggested since Dethier (1955). When a range of other monosaccharides was tested it was found that these fell into two groups: D-fructose, D-fucose, and D-galactose react chiefly with the 'fructose site'; D-arabinose, L-fucose, L-arabinose, L-sorbose, D-xylose, Lglucose and D-glucose mostly with the 'glucose site'. In the disaccharides sucrose and maltose the glucose moiety predominates and both react with the 'glucose site'. High concentrations of glucose exert no specific protective effect against PCMB treatment: this supports the view that PCMB may not react at the glucose-binding site but at a different site necessary for stimulation by $\mathrm{D}$-glucose.

From a discussion of these results and the known equilibrium changes in the conformations of a single sugar in aqueous solution, Shimada et al. adopt the more precise terms 'pyranose site' and 'furanose site' (instead of 'glucose site' and 'fructose site') representing the sites which respond especially to sugars in the pyranose and furanose forms respectively. The pyranose group was further divided into two subclasses according to the presence or absence of three successive equatorial hydroxyl groups regardless of their positions.

\section{Positive control in the test tube}

from Benjamin Lewin Molecular Genetics Correspondent

TURNING on gene expression in vitro has in general proved to be more difficult than switching it off, for although several systems have been developed in which specific repression takes place in the test tube, characterisation of positive control systems has been less successful. The positive control system investigated in most detail to date is the catabolite activator protein stimulated by cyclic AMP, whose mediation is necessary for expression of several inducible and repressible operons. Although this system seems to act on the initiation of transcription, little is known about the molecular details of its activity. Several papers in the March issue of the Proceedings of the National Academy of Sciences now take the analysis of positive control in vitro a stage further in three systems.

The first positive control system to be suggested was that of the arabinose operon, in which genetic analysis implied that a repressor protein might be converted by arabinose into an inducer protein (instead of simply suffering the inactivation characteristic of negative control systems). Lee et al. now report (Proc. natn. Acad. Sci. U.S.A., 71, $634 ; 1974)$ the development of a system in which the regulator protein, activated by arabinose, and the catabolite activator protein, stimulated by cyclic AMP, are both needed for initiation of transcription. Using a template of phage $\lambda$ carrying the arabinose genes, synthesis of ara messenger RNA can be followed by hybridisation first with $\lambda$ DNA (to remove phage sequences) and then with $\lambda$ ara DNA. This system seems completely dependent on the catabolite activator protein, is stimulated by the arabinose regulator protein in the presence of arabinose, and is inhibited by fucose (an anti-inducer of the operon). These results support previous suggestions that both the regulator protein and the catabolite activator protein must join with RNA polymerase to initiate transcription. Further analysis of this system may be important in helping to define how regulator proteins may interact with each other and with DNA.

Phage lambda itself provides a system under both negative control (lambda repressor prevents expression of integrated lysogenic lambda genomes) and positive control (needed both for the establishment of lysogeny and also for lytic development). Establishment of lysogeny depends on two phage functions ( $c I I$ and $c I I I)$ in addition to the repressor coded by the $c I$ gene, and also upon host genes. One of the host functions has now been identified by Belfort and Wulff (ibid., 779), who have isolated $h f l^{-}$mutants of Escherichia coli that show very high frequencies of lysogenisation after lambda infection. Because the $h \mathrm{fl}^{-}$ mutation is recessive, it is likely that it is caused by the loss of an active $h \mathrm{fl}^{+}$ gene product, which usually prevents establishment of lysogeny. Because $\lambda$ phage DNA lacking an active $c I I I$ gene continues to achieve the same high frequency of lysogenisation in $h \mathrm{fl}^{-}$cells that is displayed by wild type $\lambda$, it is possible that the $h f l^{+}$function is usually antagonised by the $c I I I^{+}$gene product.

That the host catabolite activator system also is implicated in establishment of lysogeny is suggested by the reduction in lysogenisation frequency in mutants defective in this system. Belfort and Wulff found that whereas these host mutants display a slight reduction in frequency of lysogenisation by $\lambda$ ' phages, they show a massive reduction in lysogenisation by $c I I I^{-}$ mutants. That the catabolite activator and $h f$ systems both act in the same pathway as the cIII phage gene product is shown by the observation that the inability of catabolite activator host mutants to lysogenise $\lambda \mathrm{clll}^{-}$mutants is reversed by the introduction of an $\mathrm{hfl}^{-}$mutation. Because catabolite re- 